\title{
I Stood Up: Social Design in Practice
}

\author{
Tom Corby, Dilys Williams, Vivek Sheth, Virkein Dhar \\ Center for Research in Education, Art and Media, University of Westminster, London, UK \\ Email: corbyt@westminster.ac.uk
}

Received 15 February 2016; accepted 15 May 2016; published 18 May 2016

Copyright (C) 2016 by authors and Scientific Research Publishing Inc.

This work is licensed under the Creative Commons Attribution International License (CC BY).

http://creativecommons.org/licenses/by/4.0/

(c) (i) Open Access

\begin{abstract}
Through practice-based research, we explore how interdisciplinary design projects can function to address social issues concerning environmental and social problems. Using two case studies developed between London in the United Kingdom, and Delhi and Ahmedabad in India, we discuss the importance of engagement with the people who the design ultimately serves. Finally, we argue that design concerned with complex social problems require equally complex, multidimensional responses, informed by bodies of knowledge, practices and approaches that lie outside of traditional design approaches.
\end{abstract}

\section{Keywords}

Design, Social Design, India, United Kingdom, Practice-Based Research, Art and Design

\section{Introduction}

This paper discusses I Stood Up, an ongoing practice-based research project developed collaboratively between an interdisciplinary team of artists and designers based in London in the United Kingdom, and Delhi and Ahmedabad in India between September 2014 and April 2015. In it, we outline the research rational of our project and report back on early findings.

The research team of Corby (visual arts), Williams (fashion design and sustainability), Sheth (exhibition and spatial design), and Dhar (performance and design) was convened at the National Institute of Design (NID) in Ahmedabad in early 2014 supported by the British Council and the UK Arts and Humanities Research Council. Our project hypothesized a role for interdisciplinary design practices to act as a platform for public articulation of "real world" problems in urban settings with a particular focus on the often connected problems of environmental degradation (Aguilar, Araujo, \& Quesada-Aguilar, 2007) and gendered violence in city locales. For example, it is known that climate change amplifies existing inequalities particularly in urban environments, leading to overcrowding, lack of privacy and the collapse of regular routines and livelihood patterns; all these can result in outbreaks of social disorder. As women constitute the largest percentage of the world's poorest people, 
they are most affected by these changes which often manifest as gendered abuse (Bartlett, 2008). In the Indian context these systemic and economic issues are exacerbated by specific cultural triggers including honour crimes and other customs and beliefs that take the status of unofficial law (Joseph, 2010). In the UK, similar issues are reported by Radford and Tsutsumi (2004) and cities such as London, containing extremes of wealth and poverty, report high levels of environmental and social issues, which are often not visible in official city narratives. In the light of this we ask: 1) What role can interdisciplinary design practices take in gathering together and communicating public concerns around gendered violence? 2) Can similar approaches be applied towards the development of public platforms to discuss environmental degradation?

\section{Methodology and Approach}

Research has been methodologically framed through the application of social and ethnographic design practices. Two case studies have been developed in India and the UK, the first of these took place at Unbox Festival at the Indira Gandhi National Centre for The Arts in New Delhi, India, the second through a series of field studies and participatory events in London. We discuss these below.

Before we proceed, it will be useful to define what we mean by ethnographic and social design in the context of our project. Ethnography originates outside of the design field in anthropology, but in the last few decades has become an important tool in the development of experimental approaches to design in the broad, particularly in relationship to interface and human computer interaction problems (Blomberg, Burrell, \& Guest, 2003). We will not rehearse its full history here, but some key aspects are worth highlighting. Simonsen and Kensing (1998) for example have identified its presence in design practices in a number of forms: 1) a design team uses an ethnographic analysis of a situation carried out by social scientists as a starting point for a project, taking relevant information and data forward; 2) designers work interactively with ethnographers, in an approach which integrates design and social science methods towards a set design problem; 3) designers and ethnographers work together to analyze a given social situation and co-design solutions (disciplinary boundaries are blurred), and finally; 4) designers versed in ethnographic methods, integrate these approaches within their practice in interaction with publics or end-users to analyze a design problem at hand and co-design solutions using participatory approaches. This final example uses ethnography in the service of a larger design initiative and we argue, is less concerned with an anthropological analysis of a social group, but rather about synthesizing particular methods derived from external disciplines (e.g. ethnography) to the service of an overall design schema within the particularities of an ongoing creative process (e.g. interviews, workshops, and other modes of social engagement). Through its focus on participation and co-design, we also argue these approaches are particularly well suited to the production of novel or appropriate design solutions rooted in a social context and seeking to be inclusive of the point of view of a group or end user. Similarly, to ethnographic approaches, social design (SD) values interdisciplinary, participatory and co-design approaches (Tonkinwise, 2015). As noted by Armstrong, Bailey, Julier et al. (2014), SD differs however in its more resolutely political outlook and its applications are often non-commercial. In such terms SD often values highly contingent design processes that are both socially embedded and social in the wider sense through their engagement of publics and non-specialist audiences in the design process.

Research completed to date, has been influenced by both approaches employing a methodological purview towards design problems oriented at social issues and developed through a collaborative and interdisciplinary lens. The following section describes this in practice through discussion of the development of a prototype design project in New Delhi, that provides a platform for victims of gendered violence to articulate and narrate their experiences. It then goes on to describe a continuing design research project where interactions involving worn artifacts function as facilitation in the voicing of environmental concerns across a range of geographic locations in London. The exchange between the two projects took place through a series of virtual discussions, email exchange and the sharing of design concepts and work between the project members.

\subsection{Case Study: I Stood Up, New Delhi}

The first phase of research consisted of fieldwork documenting the experiences of women subjected to gendered violence using recorded voice and written testimony. Consultation took place with women's groups in London, Ahmedabad and New Delhi to inform this process, and throughout research participant's contributions were anonymized by removing any identifying characteristics such as names and geographic location. This initial work was broad-based, as we wanted to collate and collect first-hand experiences in order to inform a possible design process, 
whilst at the same time brokering conversation with participants on how designers might help them mitigate or otherwise provoke public debate in India around the topic. Following this an iterative development of design prototypes and visuals, and media formats were employed to visualize the experiences of the women, in conversation with them. Further interdisciplinary research into the specifics of gender-based violence also informed this process through literature review of other disciplinary areas, in particular women's studies and social science.

Our initial expectations were that traditional object-based art and design approaches might prove popular, and we had already developed some initial concept designs for sloganized t-shirts and saris that we hoped would appeal to participants as visible ways of brokering public discussion of issues. However, in conversation with participants it soon became clear that the space that our conversations had provided for them to reflect upon and narrate incidents face-to-face was valued over and above the ideas for "objects”, or object-based design approaches we had floated. In response to this a phase of iterative prototyping was initiated that shifted the focus from producing objects to formulating convivial and safe situations for sharing and encountering experiences. This phase of research culminated in the development of a hybrid of a workshop and installation developed for the Unbox Festival at the Indira Gandhi National Centre for The Arts in New Delhi, India, and at as separate event at the Faculty of Architecture at the Centre for Environment Planning \& Technology (CEPT University) in Ahmedabad between December 2014-March 2015.

The aim of this work was to encourage participants to share and reflect upon their experiences and understanding of gendered violence, and come away with an enhanced understanding of its social impact in an Indian context. Structured as a series of interconnected spaces, the first section of the installation brought together personal stories, anonymized information, and images contributed by participants and in collaboration with No Country for Women, an NGO based in India who gather real-life stories of gender-based violence through multiple digital spaces and communities. This material took the form of anonymized large-scale posters containing witness accounts and voiced first-hand narrations accessed via headphones (Figure 1). A connecting or secondary space provided an area for visitors to leave their responses in the form of notes, signs and thoughts pinned to a wall set aside for this purpose. At this point something unexpected happened, in that the initial encounter with

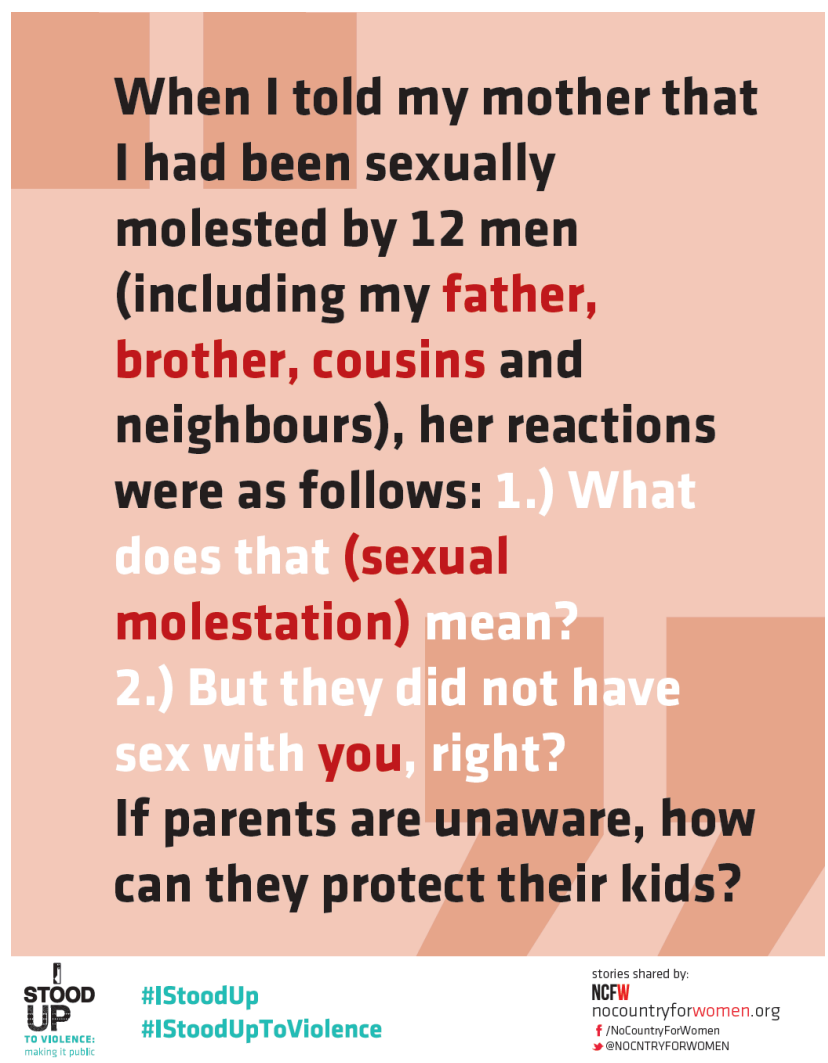

Figure 1. Example of large-scale posters containing witness accounts of gendered violence shown at the Unbox festival at the Indira Gandhi National Centre for the Arts in New Delhi, India, December 2014. 
testimonies in space one, empowered visitors to share their own experiences of gendered discrimination and violence. Thus a powerful and unplanned for reaction took place, in which visitors began to customize the installation to their own ends and needs. The final aspect of the exhibit created an interactive space that asked visitors to adapt illustrated postcards, that enabled people to imagine scenarios in which they were involved in situations involving gendered discrimination or violence or question their own responses or preconceptions of it (Figure 2). We hoped the combined effect of the installation would engage participants in a journey of encounter and reflection which they would take away with them.

\subsection{Stood Up, London}

The second phase of research took the form of fieldwork and participatory events that sought to gather data and visualize individual and collective public concerns regarding environmental degradation, carried out in four locations in London (North, South, East, West). Desk-based research informed this process, identifying the key environmental issues affecting London as a basis for further qualitative and quantitative data gathering. Again, our approach was broad-based, to collate and collect first-hand concerns and to test out a design process that might facilitate agency to voice and potentially act on concern through the wearing of a designed artifact. It should be noted that this process was not tested against an alternative to the fashion artifact, so findings are by no means generalizable. The garment as means for agency does however draw on the designer/researcher's long-standing practice, of which the message t-shirt has formed a significant part ${ }^{1}$. Design methods in this instance involved the iterative development of design prototypes including artifact, photography and questionnaire design. Throughout this research, participant's contributions were anonymized by removing any identifying characteristics such as names, and any links between verbal data gathering and photographic evidence were removed.

In this case, the artifact, a t-shirt designed to conceptually represent London as a location with the slogan "I Stood Up", created significant interest from a range of passers by, participants self-selecting to take part in the process of putting on the t-shirt, and responding to semi-structured questions about environmental concerns and being photographed (Figure 3). Participants were able to take part in one or more elements of the process, being free to opt out at any point. Here we sought to create a kind of social form through a fashion object as facilitator, and a pop-up event that encouraged individual and collective expression. It is the intertwined relationship between form and event, each propelling the other towards the creation of a spectacle in the city (Kessler, 2015), that informs urban life. In this case, the t-shirt and discourse through verbal exchange and photography offer a spectacle that demonstrates culture-making as a live social exchange in the city.

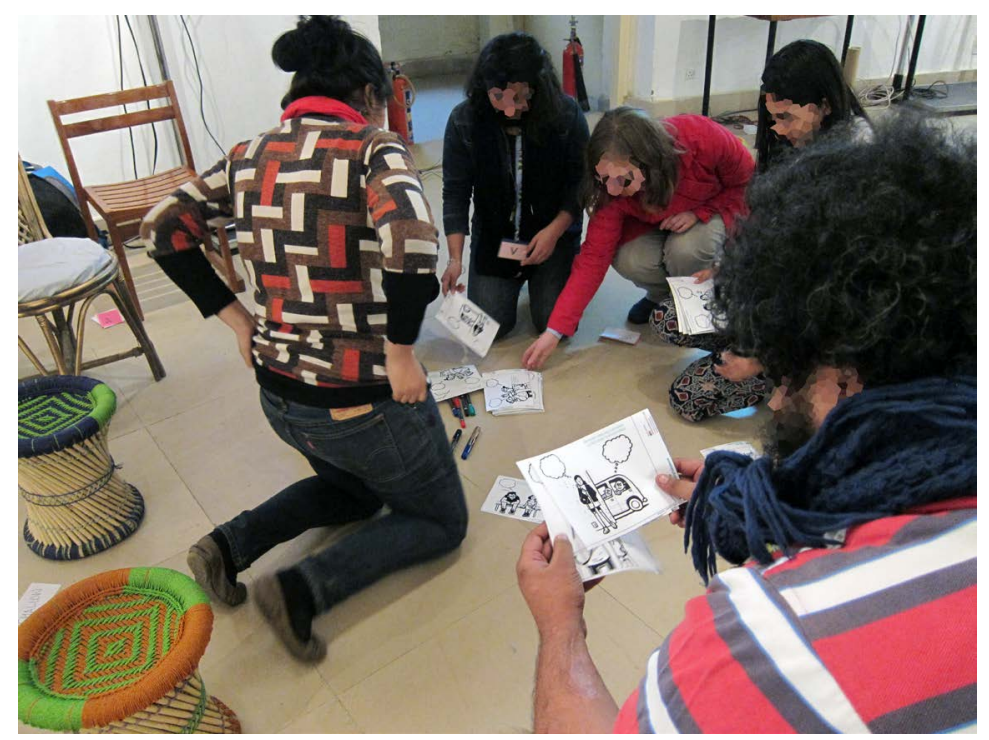

Figure 2. Documentation of workshop on gendered violence, Unbox festival at the Indira Gandhi National Centre for the Arts in New Delhi, India, December 2014.

${ }^{1}$ As head of women swear design at Katharine Hamnett (1997-2007) designing collections connecting fashion with politics. 


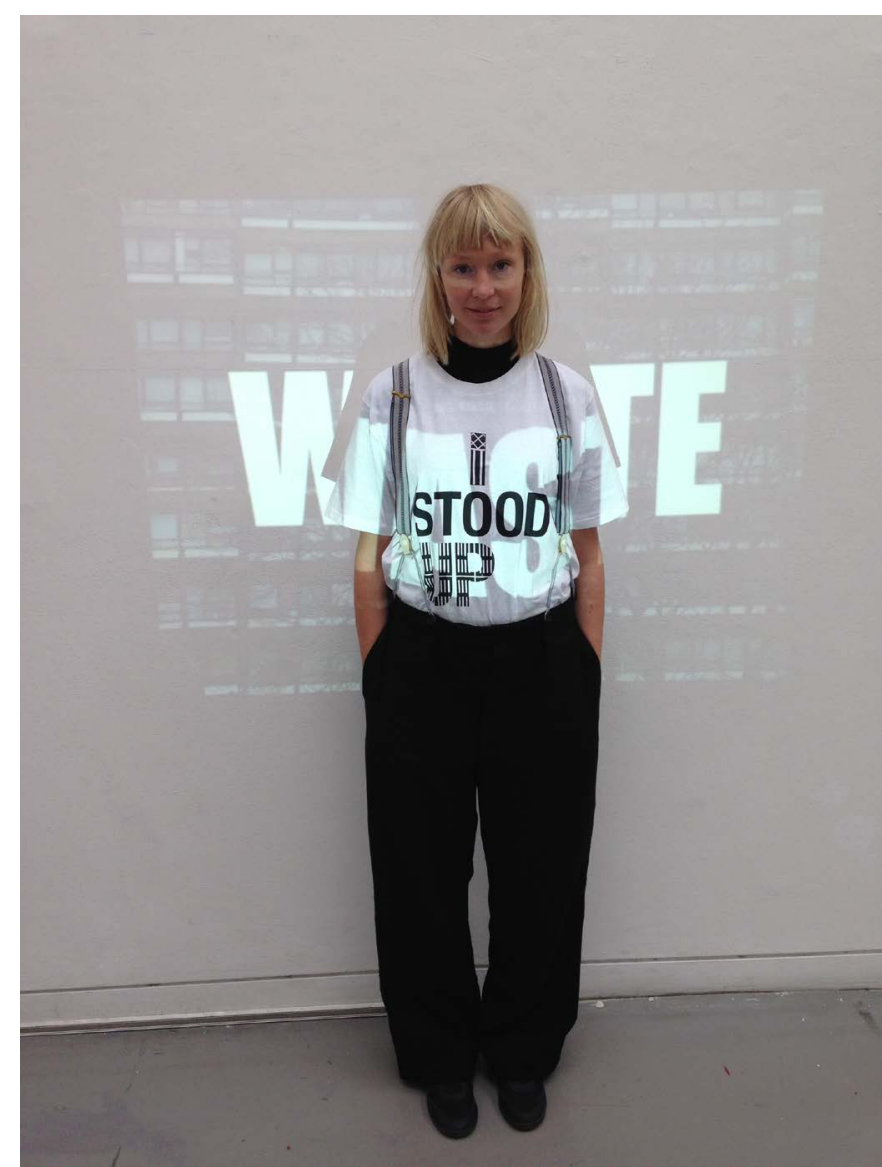

Figure 3. Workshop participants wearing I Stood Up garment, Wellcome trust, london, UK, November 2014.

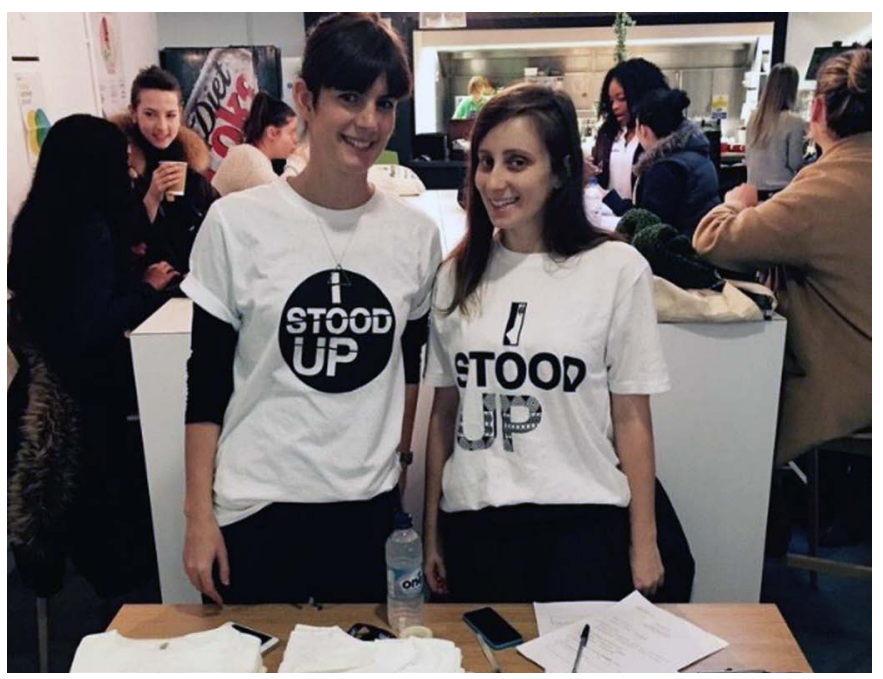

Figure 4. Workshop participants wearing I Stood Up garment, Wellcome Trust, London, UK, November 2014.

This phase of research was presented and further developed through participation in Being Human, a festival of the Humanities, where an exhibition of work, a further pop-up event and a panel discussion were held at Wellcome Trust, London, in November 2014 (Figure 4). Words, data analysis and images from the project were publicly displayed to form collective voices connected to place, as a means for individuals to consider and voice concerns relating to environmental degradation, and to begin to consider any connection between what we stand 
up in with what we stand up for. As with the "companion” project in Delhi, we hoped that through the combined effect of participating in the pop-up I Stood Up event and the discussion, participants would be able to engage in a journey of encounter and reflection which they would take away with them. The single-minded contributions of each participant (individual verbal responses and being photographed) were gathered and displayed as collective social statements in the exhibition, visualising each of us as unique whilst connected to place, community, time, culture and the city's concerns. The exhibition itself acted as a means for its audience to reflect as individuals and to consider a wider community membership.

These findings revealed the potential for the research to further develop fashion artifacts as facilitators of agency, something that is achieved in and through the engagement with a particular temporal-relational situation (Biesta and Tedder, 2006) in a city locale as part of the creation of social form in a city. Further iterations of the design prototype have been tested using artifact creation, exhibition design in indoor, publically accessible spaces, photography, text and image based publications and projection techniques. These include a co-design workshop in a committee room in the House of Lords, March 2015, participation in Cultures of Resilience, March 2015, a cross UAL exchange between researchers, tutors and students, and a second year of participation in Being Human, a festival of the Humanities, November 2015. Research to date also suggests an opportunity to develop a new strand to this exploration, that is to explore a greater understanding of the connection of individual values to the representation of fashion's social practices in garment form. The fashion sector has the ability to both shape and reflect social practice; it represents the tenor of urban life like no other medium (Breward, 2003). However, its current contribution overall, not withstanding some areas of good practice, involves significant environmental degradation, which might be at odds with the concerns of its wearers. It might therefore be surmised that further research could explore the means to give voice to citizens and also explore ways to connect individual and collective environmental concerns to fashion's social practices.

\section{Conclusion and Discussion}

Our research, fieldwork and the case studies to date support a number of initial conclusions for our work. Firstly, design that engages complex subject matter of social import, requires that designers working in such contexts should be prepared to listen and let participants lead the process. This appears risky in regard to traditional or "top-down" design processes, but we believe that the benefits outweigh the dangers in terms of positive and novel outcomes. It requires working in sometimes extremely ad-hoc ways, employing highly contingent and social modes of production. Design responses to complex social problems require complex, multidimensional responses, which are also necessarily interdisciplinary in their methodological formations, as by necessity they need to be informed by bodies of knowledge, practices and approaches that lie outside of traditional design approaches. Secondly, we consider the design product in this is context to be a set of processes, possible communications, and social activities that may (case study 2) or may not be (case study 1) facilitated by a designed or co-created artifact. If, to paraphrase Tonkinwise, design is about providing "something useful" (Tonkinwise, 2015), then the "usefulness" of design in this context is how it enables connecting, sharing and storytelling to support users and participants in bringing to public attention issues of importance through articulation of their own concerns and experiences. For us, the most effective approach to supporting this social activity as evidenced by this research is through the designed provision of participatory, social and spatial multi-sensory contexts and platforms. Cities have the capability of providing something for everybody, only because, and only when, they are created by everybody (Jacobs, 1961). That is not to say the material object of design is not significant, but rather that it functions as part of wider set of processes towards the development of social relations that places people at the centre of the design process, by interweaving place-making and form-making as a means for communities to contribute to social and environmental restorative practices.

\section{Acknowledgements}

We would like to thank the UK Arts and Humanities Research Council and the British Council for providing the funding to carry out this research.

\section{References}

Aguilar, L., Araujo, A., \& Quesada-Aguilar, A. (2007). Gender and Climate Change_Women as Agents of Change. Climate 
Change Briefing. Fact Sheet IUCN. Gland: IUCN.

Armstrong, L., Bailey, J., Julier, G., \& Kimbell, K. (2014). Social Design Futures: HEI Research and the AHRC. University of Brighton and Victoria and Albert Museum.

Bartlett, S. (2008). Climate Change and Urban Children: Implications for Adaptation in Low and Middle Income Countries. London: International Institute for Environment and Development.

Biesta, G., \& Tedder, M. (2006). How Is Agency Possible? Towards an Ecological Understanding of Agency-as-Achievement. Working Paper 5.

http://www.tlrp.org/project\%20sites/LearningLives/papers/working_papers/Working_paper_5_Exeter_Feb_06.pdf

Blomberg, J., Burrell, M., \& Guest, G. (2003). An Ethnographic Approach to Design. The Human-Computer Interaction Handbook: Fundamentals, Evolving Technologies and Emerging Applications (pp. 964-986). London: LEA.

Breward, C. (2003). Fashion. Oxford: Oxford University Press.

Jacobs, J. (1961). The Death and Life of Great American Cities. Random House.

Joseph, A. (2010). Violence Is as Violence Does. Gender Violence in India: A Prajnya Report 2010. The Gender Violence Research and Information Taskforce at The Prajna Trust.

Kessler, M. (2015). The City and the Spectacle, Memories of Future Cites Workshop. Institute of Modern Languages Research, School of Advanced Studies, University of London.

Radford, L., \& Tsutsumi, K. (2004). Globalization and Violence against Women-Inequalities in Risks, Responsibilities and Blame in the UK and Japan. Women's Studies International Forum, 27, 1-12. http://dx.doi.org/10.1016/j.wsif.2003.12.008

Simonsen, J., \& Kensing, F. (1998). Make Room for Ethnography in Design! Journal of Computer Documentation, 22, 2030.

Tonkinwise, C. (2015). Is Social Design a Thing? http://www.academia.edu/11623054/Is Social Design a Thing 